\title{
A FUNCTIONAL APPROACH TO APPROXIMATIONS FOR THE INDIVIDUAL RISK MODEL
}

\author{
BY
}

\author{
SuSAN M. PitTS
}

\begin{abstract}
A functional approach is taken for the total claim amount distribution for the individual risk model. Various commonly used approximations for this distribution are considered, including the compound Poisson approximation, the compound binomial approximation, the compound negative binomial approximation and the normal approximation. These are shown to arise as zeroth order approximations in the functional set-up. By taking the derivative of the functional that maps the individual claim distributions onto the total claim amount distribution, new first order approximation formulae are obtained as refinements to the existing approximations. For particular choices of input, these new approximations are simple to calculate. Numerical examples, including the well-known Gerber portfolio, are considered. Corresponding approximations for stop-loss premiums are given.
\end{abstract}

\section{KEYWORDS}

Total claim amount distribution, compound distributions, stop-loss premiums, Fréchet derivatives.

\section{INTRODUCTION}

A key task in risk theory is the evaluation of the distribution of the aggregate claim $S$ arising from a collection of risks over a fixed time period. Historically, two main approaches to this are via the individual risk model and via the collective risk model. These are considered in Cramér (1930) who refers back to earlier work by Lundberg and others. More recent overviews appear in Klugman, Panjer and Willmot (1998) and Rolski, Schmidli, Schmidt and Teugels (1999).

\subsection{The individual risk model}

In the individual risk model, the total amount claimed is modelled as the sum of the amounts claimed on each of the separate risks. Thus if $X_{i}$ is the claim amount for the $i$ th risk, $i=1, \ldots, m$, then the aggregate claim is 


$$
S=X_{1}+\ldots+X_{m},
$$

where it is usually assumed that the $X_{i}$ 's are independent but not necessarily identically distributed. have

If $G_{i}$ is the distribution function of $X_{i}$, so that $G_{i}(x)=P\left(X_{i} \leq x\right)$, we often

$$
G_{i}=\left(1-q_{i}\right) 1_{[0, \infty)}+q_{i} F_{i},
$$

where $q_{i} \in(0,1)$ is the probability that there is a claim on the $i$ th risk, $1_{A}$ is the indicator function of the set $A$, and $F_{i}$ is the distribution function of a positive random variable $Y_{i}$ representing the size of claim on the $i$ th risk, given that a claim occurs. Then the distribution function $F_{S}$ of the total claim amount $S$ is

$$
F_{S}=G_{1} * \ldots * G_{m},
$$

where $*$ denotes Lebesgue-Stieltjes convolution.

Example 1. A typical simple example has the distribution of $Y_{i}$ concentrated on a single point $b_{i}$, so that $X_{i}$ is the discrete random variable

$$
X_{i}= \begin{cases}0 & \text { with probability } 1-q_{i} \\ b_{i} & \text { with probability } q_{i} .\end{cases}
$$

A concrete example of this model is a group life insurance plan covering $m$ lives, where $b_{i}$ and $q_{i}$ are the amount payable on death and the mortality respectively for individual $i$ (see Klugman et al. (1998), §4.12.1).

One of the main quantities of interest in the individual model is the total claim amount distribution $F_{S}$, and we need to handle this both in terms of numerical evaluation of $F_{S}$ and also in terms of deriving theoretical properties of $F_{S}$. Although the convolution product $F_{S}=G_{1} * \ldots * G_{m}$ is a conceptually simple quantity, historically it turned out to be much easier to deal with the collective risk model, as descibed below. In addition, the use of the collective risk model in practice avoids the necessity of obtaining precise statistical estimates of each of $\mathrm{G}_{1}, \ldots, G_{m}$ from claims data.

\subsection{Approximations for the individual risk model}

In the collective risk model, we do not keep track of which risk gives rise to which claim. Instead, we model the total claim amount as the sum of a random number $N$ of claims, where the claims $Z_{1}, Z_{2}, \ldots$ are assumed to be independent, identically distributed random variables with distribution function $F_{Z}$, say, and with $\left\{Z_{i}\right\}$ assumed to be independent of $N$. Writing $T$ for the total claim amount in the collective risk model, we have

$$
T=Z_{1}+\ldots+Z_{N},
$$


with $T=0$ if $N=0$. Thus $T$ is a random sum and has a compound distribution, with distribution function $F_{T}=\sum_{k=0}^{\infty} P(N=k) F_{Z}^{\star k}$, where $F^{\star 0}=1_{[0, \infty)}$.

One popular collective model approximation to the individual model is given by a compound Poisson approximation where $N$ has a Poisson distribution with mean $\lambda$ and $F_{Z}$ is $F$, where

$$
\lambda=\sum_{i=1}^{m} q_{i} \text { and } F=\frac{1}{\lambda} \sum_{i=1}^{m} q_{i} F_{i}
$$

We use the distribution function $F_{T}$ of the resulting collective total claim amount $T$ as an approximation to the distribution function $F_{S}$ of the individual total claim amount $S$.

Another approximation uses a compound binomial approximation where $N$ has a binomial distribution with index $m$, so that

$$
N \sim \operatorname{Bin}(m, p) \text { with } p=\frac{1}{m} \sum_{i=1}^{m} q_{i}, \text { and } F_{Z}=F \text { as above. }
$$

A third collective risk model is a compound negative binomial approximation, where $N$ has a negative binomial distribution, $N \sim \mathrm{NB}(m, 1 /(1+p))$, with

$$
P(N=k)=\left(\begin{array}{c}
m+k-1 \\
m-1
\end{array}\right)\left(\frac{1}{1+p}\right)^{m}\left(\frac{p}{1+p}\right)^{k}, \quad k=0,1,2, \ldots,
$$

(so that $N$ has probability generating function $\left.G_{N}(z)=(1+p-p z)^{-m}\right)$, where $p=\sum q_{i} / m$ as in (1.2) and $F_{Z}=F$ again.

All these three approximating collective risk models have the mean of the approximating total claim amount exactly equal to the mean of the total claim amount $S$ in the individual risk model, but they all have variances that are larger than $\operatorname{var}(S)$. See Rolski et al. (1999) §4.6 and the references therein for further discussion of these approximations, and see Kuon, Radtke and Reich (1993) for different choices of parameter values in the collective risk models that ensure that both the means and the variances match those of the individual model. An alternative compound Poisson approximation has $N$ Poisson distributed with mean $\lambda^{\prime}=\sum_{i=1}^{m} q_{i} /\left(1-q_{i}\right)$ and $F_{Z}=\sum_{i=1}^{m}\left(q_{i} /\left(1-q_{i}\right)\right) F_{i} / \lambda^{\prime}$ (see Kornya (1983), and also Hipp (1986)). A third possible compound Poisson approximation has Poisson mean $\lambda^{\prime \prime}=-\sum_{i=1}^{m} \log _{e}\left(1-q_{i}\right)$ and $F_{Z}=-\sum_{i=1}^{m} \log _{e}\left(1-q_{i}\right) F_{i} / \lambda^{\prime \prime}$ (see Klugman et al (1998), §4.12.3).

All the above commonly found collective risk models have $N$ distributed as Poisson, binomial or negative binomial, and hence are such that Panjer recursion (see Panjer (1981)) can be used to calculate the compound distribution. An alternative numerical method of calculation of compound distributions is the fast Fourier transform algorithm (see Embrechts, Grübel and Pitts (1993)), whose use is not restricted to those $N$-distributions suitable for Panjer recursion. 
For moderate portfolio sizes, direct calculation methods may be useful for $F_{S}$ in the individual risk model. These direct methods include the use of generating functions, the De Pril algorithm (see De Pril (1986), (1989)), and the fast Fourier transform algorithm (see Charles (2002)). Comparing the use of the fast Fourier transform algorithm for the individual model and for the collective model, we see that in general we need $m$ transform steps for direct numerical evaluation of $F_{S}$ for the individual model (if the $F_{i}$ 's are all different), then multiplication of the $m$ transforms, and finally one inverse transform step. In contrast, for the compound Poisson approximation we need first to find $F$ in (1.1), then one transform step, then an exponentiation step and one inverse, ie fewer transforms.

A much simpler approximation is obtained by approximating $F_{S}$ by a normal distribution function (see Klugman et al. (1998) §4.12.1). If $\mu_{i}=\mathbb{E}\left(Y_{i}\right)$ and $\sigma_{i}^{2}=\operatorname{var}\left(Y_{i}\right)$ (assumed finite), then the approximating normal distribution has mean and variance given by

$$
\mu_{S}=\mathbb{E}(S)=\sum_{i=1}^{m} q_{i} \mu_{i} \text { and } \sigma_{S}^{2}=\operatorname{var}(S)=\sum_{i=1}^{m}\left(q_{i} \sigma_{i}^{2}+q_{i}\left(1-q_{i}\right) \mu_{i}^{2}\right) .
$$

This approximation is easy to calculate, although it may fail to capture important aspects, such as skewness, of $F_{S}$.

Many papers have considered approximations and calculation techniques for the individual risk model, some of which have already been mentioned. A common theme in the literature is to derive error bounds for these approximation in terms of, for example sup $\left|F_{T}(x)-F_{S}(x)\right|$ (De Pril and Dhaene (1992), Dhaene and Sundt (1997)). An important paper for our work is Hipp (1986), who gives higher order compound Poisson approximations. These are discussed below in $\S 3.1$.

\subsection{A functional approach}

In this paper, we adopt a simple functional framework that is appropriate for both the individual model and for the approximating models. In this set-up, the existing approximations described in $§ 1.2$ arise as "zeroth" order approximations, and we then refine these to obtain new naturally arising "first" order approximations which require very little extra mathematical effort beyond finding an appropriate functional framework. The approximations obtained are in principle approximation formulae, rather then numerical approximations, although they can be used to obtain numerical values.

To adopt a functional framework means that we regard the risk model as a map $\Phi$ that takes the input, for example, $\left(G_{1}, \ldots, G_{m}\right)$, onto an output quantity of interest, such as $F_{S}$. We show below that each of the collective risk and normal approximations can be written as the result of applying $\Phi$ to various input $\left(a_{1}, \ldots, a_{m}\right)$. Then, by showing that $\Phi$ is differentiable (in an appropriate sense) at $\left(a_{1}, \ldots, a_{m}\right)$, we obtain first order correction terms for the various 
approximations, giving rise to the new refined first order approximations. Higher derivatives potentially lead to higher order approximations. However, the first order ones here are particularly simple.

This functional approach has previously been used for stochastic models in renewal theory (Grübel (1989), Politis and Pitts (1998)), queueing theory (Grübel and Pitts (1992)), and ion channel models (Pitts (1998), Pitts (1999)).

The structure of the paper is as follows. In $\$ 2$ we give the functional framework for the map $\Phi$, and the relevant differentiability result. In $\S 3$, we apply the general first order correction terms to the compound Poisson, binomial and negative binomial collective risk model approximations, and also to the normal approximation. $\$ 4$ contains numerical examples, and stop-loss premiums are considered in $\S 5$. $\$ 6$ contains some miscellaneous comments.

\section{THE CONVOLUTION PRODUCT MAP}

In this section we specify the domain and codomain for the convolution product map $\Phi$, and we find its derivative. We work in the space $A$ of finite signed measures on $(\mathbb{R}, \mathcal{B})$, where $\mathcal{B}$ is the Borel $\sigma$-field of $\mathbb{R}$. We note that $A$ is contained in the space of finite complex measures on $(\mathbb{R}, \mathcal{B})$, and inherits many of the properties of this space (see Rudin (1986), chapter 6). For $a$ in $A$, we write $|a|$ for the non-negative measure given by $|a|(E)=\sup \sum_{i=1}^{\infty}\left|a\left(E_{i}\right)\right|$, the supremum being taken over all partitions $\left\{E_{i}\right\}_{i=1}^{\infty}$ of $E$ in $\mathcal{B}$, and we write $\|a\|$ for the total variation norm of $a$, given by $\|a\|=|a|(\mathbb{R})$. Then $(A,\|\cdot\|)$ is a Banach space (see Dunford and Schwartz (1958), III 7.4). The convolution of $a$ and $b$ in $A$ is $a * b$, where for $E$ in $\mathcal{B}, a * b(E)=\int a(E-x) b(d x)$, and it is easy to check that the norm inequality is satisfied, i.e. $\|a * b\| \leq\|a\|\|b\|$. Let $\delta_{b}$ be the element of $A$ that assigns a unit mass to $b \in \mathbb{R}$. Then $\delta_{0}$ is the identity element of $A$.

Now let $A^{m}=A \times \ldots \times A$, and for $\left(x_{1}, \ldots, x_{m}\right)$ in $A^{m}$, let $\left\|\left(x_{1}, \ldots, x_{m}\right)\right\|=$ $\sum_{i=1}^{m}\left\|x_{i}\right\|$. Let the convolution product map $\Phi: A^{m} \rightarrow A$ be defined by $\Phi\left(x_{1}, \ldots\right.$, $\left.x_{m}\right)=x_{1} * \ldots * x_{m}$. We sometimes write $\prod_{i=1}^{m} a_{i}$ for the convolution product $a_{1} *$ $\ldots * a_{m}$. It should be clear from the context whether $\|\cdot\|$ refers to the norm in $A$ or in $A^{m}$, and whether the symbol $\Pi$ refers to convolution of elements of $A$ or to multiplication of numbers.

We show below that $\Phi$ is Fréchet differentiable and we give its Fréchet derivative. In general, a map $\Psi: U \rightarrow B_{2}$, where $U$ is an nonempty open subset of $B_{1}$, and where $\left(B_{i},\|\cdot\|_{i}\right), i=1,2$ are Banach spaces, is Fréchet differentiable at $a \in U$ if there exists a linear bounded map $\Psi_{a}^{\prime}: B_{1} \rightarrow B_{2}$ such that for all $\varepsilon>0$, there exists $\delta>0$ such that $\|h\|_{1}<\delta$ implies

$$
\left\|\Psi(a+h)-\Psi(a)-\Psi_{a}^{\prime}(h)\right\|_{2} \leq \varepsilon\|h\|_{1} .
$$

Then $\Psi_{a}^{\prime}$ is the Fréchet derivative of $\Psi$ at $a(\S 2.1$ in Chapter 1 of Cartan (1971)).

Proposition 2. The convolution product map $\Phi$ is Fréchet differentiable at $\left(a_{1}, \ldots\right.$, $\left.a_{m}\right)$ for all $\left(a_{1}, \ldots, a_{m}\right) \in A^{m}$, with Fréchet derivative 


$$
\Phi_{\left(a_{1}, \ldots, a_{m}\right)}^{\prime}\left(h_{1}, \ldots, h_{m}\right)=\sum_{i=1}^{m} h_{i} * \prod_{j \neq i} a_{j},
$$

for $\left(h_{1}, \ldots, h_{m}\right)$ in $A^{m}$.

Proof. For $a=\left(a_{1}, \ldots, a_{m}\right)$ and $h=\left(h_{1}, \ldots, h_{m}\right)$ in $A^{m}$, we have

$$
\begin{aligned}
\left\|\Phi(a+h)-\Phi(a)-\sum_{i=1}^{m} h_{i} * \prod_{j \neq i} a_{j}\right\| & =\left\|\prod_{i=1}^{m}\left(a_{i}+h_{i}\right)-\prod_{i=1}^{m} a_{i}-\sum_{i=1}^{m} h_{i} * \prod_{j \neq i} a_{j}\right\| \\
& =\left\|\sum_{i_{1}<i_{2}} h_{i_{1}} * h_{i_{2}} * \prod_{j \neq i_{1}, i_{2}} a_{j}+\ldots+\prod_{i=1}^{m} h_{i}\right\| .
\end{aligned}
$$

This is at most

$$
\begin{aligned}
& \sum_{i_{1}<i_{2}}\left\|h_{i_{1}}\right\|\left\|h_{i_{2}}\right\| \prod_{j \neq i_{1}, i_{2}}\left\|a_{j}\right\|+\ldots+\prod_{i=1}^{m}\left\|h_{i}\right\| \\
& \leq\|h\|^{2}\left[\left(\begin{array}{c}
m \\
2
\end{array}\right)\|a\|^{m-2}+\left(\begin{array}{c}
m \\
3
\end{array}\right)\|h\|\|a\|^{m-3}+\ldots+\|h\|^{m-2}\right] .
\end{aligned}
$$

For $\|h\| \leq 1$, this is bounded above by $\|h\|^{2}(1+\|a\|)^{m}$. Given $\varepsilon>0$, for $\|h\| \leq$ $\min \left\{\varepsilon /\left((1+\|a\|)^{m}\right), 1\right\}$, we have that $(2.3)$ is bounded above by $\varepsilon\|h\|$, so that (2.1) holds, and the derivative of $\Phi$ is as in (2.2).

The map $\Phi_{a}^{\prime}$ is linear. If $\|h\| \leq 1$, then

$$
\left\|\Phi_{a}^{\prime}(h)\right\| \leq \sum_{i=1}^{m}\left\|h_{i}\right\| \prod_{j \neq i}\left\|a_{j}\right\| \leq m\|a\|^{m-1},
$$

so that $\Phi_{a}^{\prime}$ is bounded. Hence the map $\Phi$ is Fréchet differentiable at $a$ with Fréchet derivative $\Phi_{a}^{\prime}$ there.

\section{ApPlications to THE INDIVIDUAL RISK MODEL}

For the individual risk model, suppose the distribution $G_{i}$ of the $i$ th individual claim $X_{i}$ corresponds to element $x_{i}$ of $A, i=1, \ldots, m$. Then the total claim amount distribution $F_{S}$ corresponds to $s$ in $A$ given by $s=\Phi\left(x_{1}, \ldots, x_{m}\right)$. Using notation as in $\$ 1.1$, if $y_{i}$ in $A$ corresponds to the distribution of $Y_{i}$, then we set $x_{i}=\left(1-q_{i}\right) \delta_{0}+q_{i} y_{i} \in A, i=1, \ldots, m$. For Example 1 we have $x_{i}=\left(1-q_{i}\right) \delta_{0}+$ $q_{i} \delta_{b_{i}} \in A, i=1, \ldots, m$.

We approximate $\Phi\left(x_{1}, \ldots, x_{m}\right)$ by $\Phi\left(a_{1}, \ldots, a_{m}\right)$ where $a_{i} \in A, i=1, \ldots, m$, and the $a_{i}$ 's are chosen so that $\Phi\left(a_{1}, \ldots, a_{m}\right)$ is easily found. We call this the "zeroth" order approximation. We refine this by adding a first order correction term 


$$
\Phi_{\left(a_{1}, \ldots, a_{m}\right)}^{\prime}\left(x_{1}-a_{1}, \ldots, x_{m}-a_{m}\right)=\sum_{i=1}^{m}\left(x_{i}-a_{i}\right) * \prod_{j \neq i} a_{j},
$$

which leads to the first order approximation

$$
\Phi\left(x_{1}, \ldots, x_{m}\right) \approx \Phi\left(a_{1}, \ldots, a_{m}\right)+\sum_{i=1}^{m}\left(x_{i}-a_{i}\right) * \prod_{j \neq i} a_{j} .
$$

If all the $a_{i}$ 's are the same, and equal to $a$ say, then the zeroth order approximation is $a^{* m}$. The first order correction term is then

$$
\Phi_{(a, \ldots, a)}^{\prime}\left(x_{1}-a, \ldots, x_{m}-a\right)=\left(\sum_{i=1}^{m} x_{i}\right) * a^{*(m-1)}-m a^{* m},
$$

and the first order approximation is

$$
\Phi\left(x_{1}, \ldots, x_{m}\right) \approx\left(\sum_{i=1}^{m} x_{i}\right) * a^{*(m-1)}-(m-1) a^{* m} .
$$

We apply these results to the various approximations introduced in $\S 1.2$. For each case, we show how the approximation can be written in the form of a zeroth order approximation $\Phi\left(a_{1}, \ldots, a_{m}\right)$, for suitably chosen $a_{i}$ 's, and then we find the form of the resulting first order approximation.

\subsection{The compound Poisson approximation}

We consider the compound Poisson approximation given in (1.1), with $\lambda=$ $\sum_{i=1}^{m} q_{i}$ and $F_{Z}=F=\left(\sum_{i=1}^{m} q_{i}\right)^{-1} \sum_{i=1}^{m} q_{i} F_{i}$. Given the relative simplicity of (3.3) compared to (3.1), we approximate the compound Bernoulli distribution $x_{i}=$ $\left(1-q_{i}\right) \delta_{0}+q_{i} y_{i}$ by a compound Poisson distribution $a$ with Poisson parameter $\sum_{i=1}^{m} q_{i} / m$ and with claims size distribution $y$ in $A$ given by $y=\left(\sum_{i=1}^{m} q_{i}\right)^{-1}$ $\sum_{i=1}^{m} q_{i} y_{i}$ (so that $y$ corresponds to $F$ in (1.1)). Then $a^{* m}$ is an approximation to $x_{1} * \ldots * x_{m}$, and moreover, $a^{* m}$ is the convolution of compound Poisson distributions and hence is itself a compound Poisson distribution, with Poisson parameter $\lambda=\sum_{i=1}^{m} q_{i}$ and the claim size distribution $y$, so that $a^{* m}$ is the compound Poisson approximation in (1.1). Thus the approximation in (1.1) is $\Phi(a$, $\ldots, a)$, and so fits into our functional framework. We note that the compound Poisson approximation can be written as $\exp \left(\lambda\left(y-\delta_{0}\right)\right)$ in $A$, where for $a \in \mathrm{A}$, $\exp a=\sum_{k=0}^{\infty} a^{* k} / k$ !, so that the above relationship says that $\exp \left(\lambda\left(y-\delta_{0}\right)\right)=$ $\left(\exp \left(\lambda\left(y-\delta_{0}\right) / m\right)\right)^{* m}$.

We apply (3.3) to obtain the first order approximation 


$$
\begin{aligned}
& \left(\sum_{i=1}^{m} x_{i}\right) * a^{*(m-1)}-(m-1) a^{* m} \\
& =\left[\left(\sum\left(1-q_{i}\right)\right) \delta_{0}+\left(\sum q_{i} y_{i}\right)\right] * a^{*(m-1)}-(m-1) a^{* m} \\
& =(m-\lambda) a^{*(m-1)}+\lambda y * a^{*(m-1)}-(m-1) a^{* m} .
\end{aligned}
$$

The convolution product $a^{*(m-1)}$ is a compound Poisson distribution with Poisson parameter $\left(1-m^{-1}\right) \sum q_{i}$ and claim size distribution $y$. If the zeroth order approximation $a^{* m}$ has already been found, then the first order correction term involves evaluation of the compound Poisson distribution $a^{*(m-1)}$ (which is as easy to find as the zeroth order approximation) and it also involves evaluation of one potentially difficult convolution $\lambda y * a^{*(m-1)}$. Roughly, writing $\operatorname{comp}(\operatorname{Poiss}(\lambda), F)$ to denote a compound Poisson distribution with Poisson parameter $\lambda$ and claim size distribution $F$, we obtain,

$$
\begin{aligned}
F_{S} \approx & \left(m-\sum q_{i}\right) \operatorname{comp}(\operatorname{Poiss}((m-1) \lambda / m), F) \\
& +\left(\sum q_{i} F_{i}\right) * \operatorname{comp}(\operatorname{Poiss}((m-1) \lambda / m), F) \\
& -(m-1) \operatorname{comp}(\operatorname{Poiss}(\lambda), F) .
\end{aligned}
$$

For illustration purposes only, we can apply this expression to Example 1 where $F_{i}=\delta_{b_{i}}$, although we note that in this case exact calculation of $s$ is avaiable via De Pril recursion (De Pril (1986)). Write $s_{k}$ for $P(S=k$ ), and write comp (Poiss $(\lambda), F)_{k}$ for the probability that the given compound Poisson random variable takes the value $k$. Then

$$
\begin{aligned}
s_{k} \approx & \left(m-\sum q_{i}\right) \operatorname{comp}(\operatorname{Poiss}((m-1) \lambda / m), F)_{k} \\
& +\sum q_{i} \operatorname{comp}(\operatorname{Poiss}((m-1) \lambda / m), F)_{k-b_{i}} \\
& -(m-1) \operatorname{comp}(\operatorname{Poiss}(\lambda), F)_{k} .
\end{aligned}
$$

In terms of numerical calculation via transforms, we suppose we already have the fast Fourier transform of $y$ and $a^{* m}$ in yfft and zerofft respectively. To calculate the first order approximation in (3.4), we need basically one exponentiation step and one multiplication step to find

$$
((m-\lambda)+\lambda \mathrm{yfft}) \exp ((m-1) \lambda / m(\mathrm{yfft}-1))-(m-1) \text { zerofft },
$$

and then one inverse transform.

An alternative representation of the zeroth order compound Poisson approximation as a convolution product is to approximate the compound Bernoulli factor $x_{i}=\left(1-q_{i}\right) \delta_{0}+q_{i} y_{i}$ by the compound Poisson distribution $a_{i}$, with Poisson parameter $q_{i}$ and with claim size distribution $y_{i}$. Then $a_{1} * \ldots * a_{m}$ is the same zeroth order compound Poisson distribution given in (1.1). The first order approximation is given by (3.1). Although each $\prod_{j \neq i} a_{j}$ is a compound Poisson 
distribution, it has a different Poisson parameter and a different claim size distribution for each $i$. Thus this first order approximation is more complicated to evaluate than (3.4). For numerical evaluation via transforms, this would need $m$ different exponentiations and $m$ different counterparts of yfft.

The same procedure can be applied to the other compound Poisson approximations in $\$ 1.2$ to get first order approximations.

Hipp (1986) obtains first and higher order approximations, together with error bounds, for compound Poisson approximations. Each of these approximations is itself a (generalised) compound Poisson measure. For the zeroth order compound Poisson approximation in (1.1), Hipp's corresponding next higher order approximation in our notation is the element $h^{(1)}$ of $A$ given by

$$
h^{(1)}=\exp \left(\lambda^{(1)}\left(y^{(1)}-\delta_{0}\right)\right)
$$

where

$$
\begin{aligned}
& \lambda^{(1)}=\sum_{i=1}^{m}\left(q_{i}+\frac{q_{i}^{2}}{2}\right), \\
& y^{(1)}=\frac{1}{\lambda^{(1)}} \sum_{i=1}^{m}\left(\left(q_{i}+q_{i}^{2}\right) y_{i}-\frac{1}{2} q_{i}^{2} y_{i}^{* 2}\right) .
\end{aligned}
$$

This is different from the first order approximations we obtained above. While (3.5) turns out to be much more accurate than (3.3) in the examples considered below in $\S 4$, we note that it is intrinsically more complicated than our approximations. Calculation of (3.6) involves computation of $y_{i}^{* 2}$ for $i=1, \ldots, m$. For some distributions this is easy, for example if $y_{i}=\delta_{b_{i}}$ then $y_{i}^{* 2}=\delta_{2 b_{i}}$, but for general $y_{i}$ 's, calculation via fast Fourier transform methods involves finding the transform of each $y_{i}$ and squaring it. In summary, the first order approximation in (3.3) is cruder but often simpler than Hipp's corresponding approximation. Furthermore, our approximation technique is also applied to compound binomial, compound negative binomial and normal approximations below, whereas Hipp's more accurate expansions are only for compound Poisson approximations. Similar comments apply to the expansions discussed in Čekanavičius and Wang (2003).

\subsection{The compound binomial approximation}

The compound binomial approximation given in (1.2) approximates $x_{1} * \ldots * x_{m}$ by $\left((1-p) \delta_{0}+p y\right)^{* m}$, where $p=\sum q_{i} / m$ and $y=\sum q_{i} y_{i} / \sum q_{i}$. It is immediate that this approximation is an $m$-fold convolution product and that it fits into our functional framework. This compound binomial approximation uses the same compound Bernoulli distribution $a=(1-p) \delta_{0}+p y$ to approximate each compound Bernoulli factor $x_{i}$, so that $x_{1} * \ldots * x_{m}$ is approximated by $a^{* m}$.

We note that 


$$
\begin{aligned}
\frac{1}{m} \sum_{i=1}^{m} x_{i} & =\frac{1}{m} \sum_{i=1}^{m}\left(1-q_{i}\right) \delta_{0}+\frac{1}{m} \sum_{i=1}^{m} q_{i} y_{i} \\
& =(1-p) \delta_{0}+p y \\
& =a .
\end{aligned}
$$

Hence, by (3.2), the first order correction term for the compound binomial approximation is

$$
\left(\left(\sum_{i=1}^{m} x_{i}\right)-m a\right) * a^{*(m-1)}=0,
$$

and so in this case the zeroth and first order approximations are the same.

\subsection{The compound negative binomial approximation}

The compound negative binomial approximation in (1.3) has moment generating function

$$
M_{T}(t)=\left(1+p-p M_{Y}(t)\right)^{-m},
$$

where $M_{Y}(t)$ is the moment generating function of a random variable with distribution function $F$. Hence $M_{T}(t)=a(t)^{m}$, where $a(t)=\left(1+p-p M_{Y}(t)\right)^{-1}$ is the moment generating function of a compound geometric distribution. This compound geometric distribution corresponds to an element $a$, say, of $A$. Hence the compound negative binomial approximation can be written as the output $a^{* m}$ of the convolution product map.

Using (3.2), the first order correction term is

$$
\left(\left(m-\sum q_{i}\right) \delta_{0}+\left(\sum q_{i}\right) y\right) * a^{*(m-1)}-m a^{* m},
$$

and $a^{*(m-1)}$ here is a compound negative binomial distribution with $N \sim$ NB $(m-1,1 /(p+1))$ and claim size distribution $y$. Thus this expression is relatively easy to evaluate with only one potentially difficult convolution.

\subsection{The normal approximation}

We apply the above technique to the Normal approximation in $\S 1.2$. Write $f\left(\mu, \sigma^{2}\right)$ for a $N\left(\mu, \sigma^{2}\right)$ distribution with mean $\mu$ and variance $\sigma^{2}$. Then, taking $a_{i}$ to be $f\left(\mathbb{E}\left(X_{i}\right)\right.$, var $\left.\left(X_{i}\right)\right)$, where $\mathbb{E}\left(X_{i}\right)=q_{i} \mu_{i}=\mu_{X_{i}}$ say, and $\operatorname{var}\left(X_{i}\right)=q_{i} \sigma_{i}^{2}+q_{i}\left(1-q_{i}\right)$ $\mu_{i}^{2}=\sigma_{X_{i}}^{2}$ say, we have the zeroth order approximation $\Phi\left(a_{1}, \ldots, a_{m}\right)=f\left(\mu_{S}, \sigma_{S}^{2}\right)$, ie the usual normal approximation to the distribution of $S$. We write $\mu_{\backslash i}$ for $\sum_{j \neq i} \mu_{X_{j}}$ and $\sigma_{\downarrow i}^{2}$ for $\sum_{j \neq i} \sigma_{X_{j}}^{2}$, and obtain from (3.1) the first order approximation 


$$
s=\Phi\left(x_{1}, \ldots, x_{m}\right) \approx \sum_{i=1}^{m} x_{i} * f\left(\mu_{\backslash_{i}}, \sigma_{\downarrow_{i}}^{2}\right)-(m-1) f\left(\mu_{S}, \sigma_{S}^{2}\right) .
$$

As we have seen for compound distribution approximations, simplifications often occur when $a_{1}=\ldots=a_{m}=a$, say. If we take $a=f\left(\mu_{S} / m, \sigma_{S}^{2} / m\right)$, then the zeroth order approximation $\Phi(a, \ldots, a)=a^{* m}$ corresponds to the same Normal approximation $N\left(\mu_{S}, \sigma_{S}^{2}\right)$ in (1.4). The first order approximation is

$$
s \approx\left(\sum_{i=1}^{m} x_{i}\right) * f\left(\frac{m-1}{m} \mu_{S}, \frac{m-1}{m} \sigma_{S}^{2}\right)-(m-1) f\left(\mu_{S}, \sigma_{S}^{2}\right) .
$$

The above two approaches to the Normal approximation give the same zeroth order approximations, but different first order approximations. The expression (3.8) is easier to evaluate, since it has just one potentially awkward convolution $\left(\sum_{i=1}^{m} x_{i}\right) * f\left(\left(1-m^{-1}\right) \mu_{S},\left(1-m^{-1}\right) \sigma_{S}^{2}\right)$, whereas (3.7) has $m$ such convolutions.

If we apply (3.7) and (3.8) to Example 1 we obtain respectively the simple approximation formulae

$$
s \approx \sum_{i=1}^{m}\left(1-q_{i}\right) f\left(\mu_{\backslash i}, \sigma_{\backslash i}^{2}\right)+\sum_{i=1}^{m} q_{i} f\left(\mu_{\backslash i}+b_{i}, \sigma_{\backslash i}^{2}\right)-(m-1) f\left(\mu_{S}, \sigma_{S}^{2}\right),
$$

and

$$
\begin{aligned}
s \approx & \left(\sum_{i=1}^{m}\left(1-q_{i}\right)\right) f\left(\left(1-m^{-1}\right) \mu_{S},\left(1-m^{-1}\right) \sigma_{S}^{2}\right) \\
& +\sum_{i=1}^{m} q_{i} f\left(\left(1-m^{-1}\right) \mu_{S}+b_{i},\left(1-m^{-1}\right) \sigma_{S}^{2}\right)-(m-1) f\left(\mu_{S}, \sigma_{S}^{2}\right) .
\end{aligned}
$$

\section{NumericAl EXAMPLES}

Table 1 gives Gerber's portfolio of 31 policies (page 35 in Gerber (1979); see also Hipp (1986), Kuon et al. (1993)). Here, each $x_{i}$ is of the form $x_{i}=\left(1-q_{i}\right) \delta_{0}+$ $q_{i} \delta_{b_{i}}$, as in Example 1.

TABLE 1

GERBER'S PORTFOLIO OF 31 POLICIES

\begin{tabular}{cc|ccccc}
\hline \hline & $\begin{array}{c}\text { No. of } \\
\text { policies }\end{array}$ & $\mathbf{1}$ & $\mathbf{2}$ & $\mathbf{3}$ & $\mathbf{4}$ & $\mathbf{5}$ \\
\hline \multirow{4}{*}{$q$} & 0.03 & 2 & 3 & 1 & 2 & 0 \\
& 0.04 & 0 & 1 & 2 & 2 & 1 \\
& 0.05 & 0 & 2 & 4 & 2 & 2 \\
& 0.06 & 0 & 2 & 2 & 2 & 1 \\
\hline \hline
\end{tabular}


TABLE 2

ZEROTH ORDER $S^{(0)}$ AND FIRST ORDER $S^{(1)}$ APPROXIMATIONS FOR GERBER PORTFOLIO. $s$ : TRUE TOTAL CLAIM AMOUNT DISTRIBUTION; SUFFIX $p$ DENOTES COMPOUND POISSON, $b$ DENOTES COMPOUND BINOMIAL AND $n b$ DENOTES COMPOUND NEGATIVE BINOMIAL.

\begin{tabular}{rllllll}
\hline \hline & \multicolumn{1}{c}{$s$} & \multicolumn{1}{c}{$s_{p}^{(0)}$} & \multicolumn{1}{c}{$s_{p}^{(1)}$} & $s_{b}^{(0)}=s_{b}^{(1)}$ & \multicolumn{1}{c}{$s_{n b}^{(0)}$} & \multicolumn{1}{c}{$s_{n b}^{(1)}$} \\
\hline 0 & 0.238195 & 0.246597 & 0.238563 & 0.238688 & 0.254283 & 0.238206 \\
1 & 0.0147337 & 0.0147958 & 0.0150128 & 0.0149986 & 0.0145977 & 0.0150528 \\
2 & 0.0877342 & 0.0867528 & 0.0880305 & 0.0879481 & 0.0855859 & 0.0882629 \\
3 & 0.113183 & 0.111224 & 0.112917 & 0.112820 & 0.109672 & 0.113193 \\
4 & 0.110709 & 0.110397 & 0.112271 & 0.112029 & 0.108658 & 0.112466 \\
5 & 0.0963274 & 0.0928590 & 0.0947189 & 0.0947052 & 0.0911054 & 0.0947924 \\
6 & 0.0615487 & 0.0610080 & 0.0625437 & 0.0625913 & 0.0595251 & 0.0624119 \\
7 & 0.0690221 & 0.0654270 & 0.0669503 & 0.0670024 & 0.0639431 & 0.0668063 \\
8 & 0.0548171 & 0.0545768 & 0.0556304 & 0.0556748 & 0.0535273 & 0.0555076 \\
9 & 0.0431471 & 0.0413208 & 0.0418356 & 0.0418689 & 0.0407741 & 0.0417435 \\
10 & 0.0301073 & 0.0305794 & 0.0306723 & 0.0306936 & 0.0304320 & 0.0306124 \\
11 & 0.0235292 & 0.0233079 & 0.0231400 & 0.0231499 & 0.0234149 & 0.0231106 \\
12 & 0.0182824 & 0.01834380 & 0.0180375 & 0.0180376 & 0.0185947 & 0.0180345 \\
13 & 0.0125093 & 0.0131494 & 0.0127405 & 0.0127325 & 0.0135121 & 0.0127596 \\
14 & 0.00871076 & 0.00921800 & 0.00876679 & 0.00875461 & 0.00963364 & 0.00879785 \\
15 & 0.00591165 & 0.00650426 & 0.00606548 & 0.00605269 & 0.00691867 & 0.00609903 \\
16 & 0.00415190 & 0.00459553 & 0.00420229 & 0.00419105 & 0.00497493 & 0.00423258 \\
17 & 0.00271505 & 0.00317641 & 0.00284151 & 0.00283267 & 0.00350619 & 0.00286608 \\
18 & 0.00174094 & 0.00212341 & 0.00184783 & 0.00184149 & 0.00240025 & 0.00186613 \\
19 & 0.00111736 & 0.00141386 & 0.00119392 & 0.00118991 & 0.00163906 & 0.00120617 \\
\hline \hline
\end{tabular}

Our approach allows us to find the corresponding first order correction terms for each of the compound Poisson, compound binomial and compound negative binomial approximations given in (1.1), (1.2) and (1.3) respectively. Table 2 shows the true $s$, the compound Poisson zeroth and first order approximations $s_{p}^{(0)}$ and $s_{p}^{(1)}$, the compound binomial zeroth order approximation $s_{b}^{(0)}$ (this is the same as the first order approximation, see §3.2), and the zeroth and first order compound negative binomial approximations $s_{n b}^{(0)}$ and $s_{n b}^{(1)}$. For each approximation, Table 2 shows the mass given to $k=0,1, \ldots, 19$. These are all calculated using the fast Fourier transform.

The $\|\cdot\|$ errors for these approximations are given in the first row of Table 3. For an approximation $s^{(i)}$, write $S^{(i)}$ for the mass given by this approximation to the interval $(-\infty, x]$. The second row of Table 3 gives $\sup _{x}\left|S^{(i)}(x)-F_{S}(x)\right|$. Our theory only concerns $\|\cdot\|$-errors, but we include the sup-error since other papers (in particular Hipp (1986)) refer to this distance measure.

From Table 3, we see that for Poisson and negative binomial cases, going from zeroth to first order approximations leads to a reduction in both types of error. We see that the compound binomial is the best of the zeroth order approximations for both types of error. Further, the compound Poisson and compound negative binomial first order approximations are similar to each 
TABLE 3

GERBER PORTFOLIO: ERRORS FOR THE ZEROTH AND FIRST ORDER COMPOUND POISSON, COMPOUND BINOMIAL AND COMPOUND NEGATIVE BINOMIAL APPROXIMATIONS

\begin{tabular}{cc|c|c|c|c}
\cline { 2 - 6 } & \multicolumn{2}{c|}{ Poisson } & Binomial & \multicolumn{2}{c}{ Negative Binomial } \\
\cline { 2 - 6 } & zeroth & first & zeroth/first & zeroth & first \\
\hline$\|\cdot\|$-error & 0.0263 & 0.0118 & 0.0118 & 0.0479 & 0.0117 \\
sup-error & 0.0084 & 0.0022 & 0.0021 & 0.0161 & 0.0026 \\
\hline \hline
\end{tabular}

other and also similar to the zeroth order (= first order) compound binomial approximation, for both errors. For $\|\cdot\|$-error, the compound negative binomial first order approximation is slightly better than the other two, whereas for sup-error, the compound binomial zeroth order is slightly better than the other two first order approximations. Table 2 shows that these general conclusions do not necessarily hold for each $k$-value.

We compare our approximations with Hipp's first order approximation $h^{(1)}$, which is considered for this Gerber portfolio in Hipp (1986). We recall from $\$ 3.1$ that $h^{(1)}$ is intrinsically more complicated than our rather simple first order approximation, in that $h^{(1)}$ requires $y_{i}^{* 2}$ for each $i=1, \ldots, m$. As might be expected, $h^{(1)}$ is a much more accurate appproximation, with $\|\cdot\|$-error 0.0017 and sup-error 0.000295 .

The Gerber 100 portfolio is obtained from the above portfolio by multiplying all the numbers in the body of Table 1 by 100, resulting in a portfolio of 3100 policies (see Kuon et al. (1993)). The errors for the various approximations for this portfolio are given in Table 4 . We see again that the first order approximations are improvements on the zeroth order ones. For this example, the zeroth order compound binomial approximation is better than all others in the table for $\|\cdot\|$-error, but for sup-error, both the first order compound Poisson and negative binomial approximations improve on the zeroth order compound binomial. Hence, as a general conclusion from these two examples, perhaps it is worth considering (a) compound binomial approximations, and (b) the simple first order approximations for the compound Poisson and the compound negative binomial.

For the Gerber 100 portfolio, we see again, as expected, that the Hipp first order approximation does very much better, with $\|\cdot\|$-error 0.00013 and sup-error 0.000017 .

TABLE 4

GERBER 100 PORTFOLIO: ERRORS FOR THE ZEROTH AND FIRST ORDER COMPOUND POISSON, COMPOUND BINOMIAL AND COMPOUND NEGATIVE BINOMIAL APPROXIMATIONS

\begin{tabular}{c|c|c|c|c|c}
\cline { 2 - 6 } & \multicolumn{2}{|c|}{ Poisson } & Binomial & \multicolumn{2}{c}{ Negative Binomial } \\
\cline { 2 - 6 } & zeroth & first & zeroth/first & zeroth & first \\
\hline$\|\cdot\|$-error & 0.0244 & 0.00481 & 0.00439 & 0.0435 & 0.00611 \\
sup-error & 0.0063 & 0.0012 & 0.0011 & 0.0112 & 0.0016 \\
\hline \hline
\end{tabular}


TABLE 5

EXAMPLE FROM KLUGMAN ET $A L$.

\begin{tabular}{c|ccc}
\hline \hline Age group & No. of policies & $\boldsymbol{q}_{\boldsymbol{i}}$ & $\boldsymbol{b}_{\boldsymbol{i}}$ \\
\hline $18-35$ & 400 & 0.03 & 5 \\
$36-50$ & 300 & 0.07 & 3 \\
$51-65$ & 200 & 0.10 & 2 \\
\hline \hline
\end{tabular}

Rolski et al. (page 144) compare the (zeroth order) compound Poisson, compound binomial and compound negative binomial approximations given in (1.1), (1.2) and (1.3) respectively for a different small portfolio of 29 policies, and they find that, in terms of relative error, the compound binomial is better than the compound Poisson, which is in turn better than the compound negative binomial. This is in line with the results for the zeroth order approximations for the two examples above. They also find that Hipp's first and second order approximations give much smaller relative errors.

For an example involving continuous random variables, we consider an example from Klugman et al. (1998), page 373, of a portfolio of 900 policies which divide into three groups, where for an individual in the $i$ th group, the claim amount distribution is $x_{i}=\left(1-q_{i}\right) \delta_{0}+q_{i} y_{i}$, where $y_{i}$ corresponds to an exponential distribution with mean $b_{i}$, see Table 5 .

The true total claim amount distribution has a mass at zero and a density over $(0, \infty)$. The densities for the true total claim amount distribution, the zeroth order normal approximation, and the first order normal approximation in (3.8) are shown in Figure 1. The first order approximation does a better job than the zeroth order approximation of capturing the shape of the true density. For the zeroth order approximation, the $\|\cdot\|$ and sup-errors are 0.088 and 0.023 respectively, and the corresponding errors for the first order approximation are 0.022 and 0.0039 .

\section{STOP-LOSS PREMIUMS}

In considering compound distribution approximations for the individual model, several authors also compare the resulting approximations for stop-loss premiums (for example, Hipp (1986), Kuon et al. (1993), De Pril and Dhaene (1992), Dhaene and Sundt (1997)). In this section, we extend our functional approach to stop-loss premiums, and we obtain new first order approximations that are refinements of the existing approximations in the literature.

In this section we restrict for simplicity to non-negative random variables and we consider elements of $A$ that are concentrated on $[0, \infty)$. For a non-negative total claim amount $S$ with distribution function $F_{S}$ we suppose $\mathbb{E}(S)<\infty$. Then the stop-loss premium with retention $y(>0)$ is

$$
\mathbb{E}\left((S-y)_{+}\right)=\int_{y}^{\infty}(x-y) F_{S}(d x)=\int_{y}^{\infty}\left(1-F_{S}(x)\right) d x,
$$




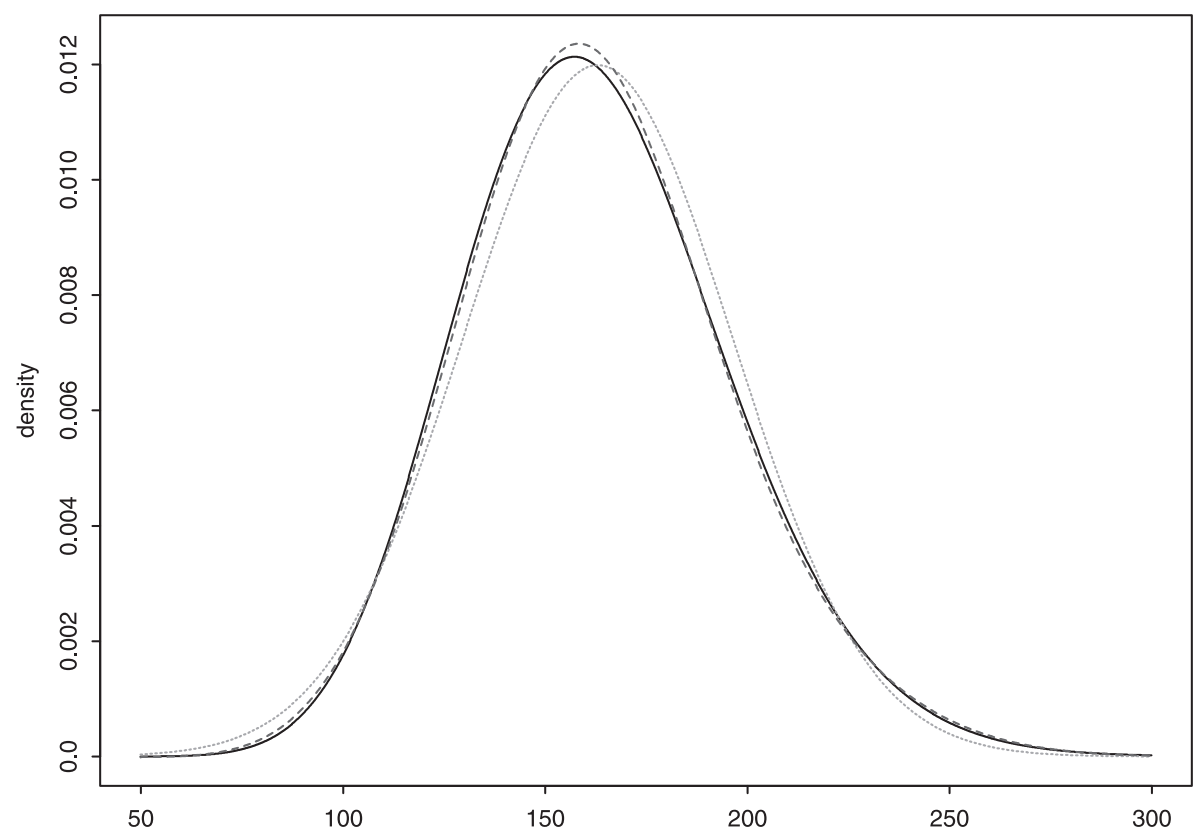

FIGURE 1. True (solid line), zeroth order normal approximation (dotted line) and first order normal approximation (broken line) for the example from Klugman et al.

where $x_{+}=\max \{x, 0\}$. This last expression motivates our choice of functional, but first we define a relevant space.

Let $A_{1}=\left\{a \in A: a\right.$ is concentrated on $\left.[0, \infty), \int t|a|(d t)<\infty\right\}$, where $|a|$ is as in $\S 2$. For $a$ in $A_{1}$, put $\|a\|_{1}=\int(1+t)|a|(d t)=\|a\|+\int_{0}^{\infty}|a|((t, \infty)) d t$. It is readily checked that $\left(A_{1},\|\cdot\|_{1}\right)$ is a Banach space, and that for $a, b \in A_{1}$, their convolution product $a * b$ is also in $A_{1}$, with $\|a * b\|_{1} \leq\|a\|_{1}\|b\|_{1}$. For $\left(a_{1}, \ldots, a_{m}\right)$ in $A_{1}^{m}$, we have that $\Phi\left(a_{1}, \ldots, a_{m}\right)=a_{1} * \ldots * a_{m}$ is in $A_{1}$.

For $a$ in $A_{1}$ and $y>0$, define $T_{y}(a)$ by

$$
T_{y}(a)=\int_{y}^{\infty} a((t, \infty)) d t .
$$

The condition that $a$ is in $A_{1}$ ensures that $\left|T_{y}(a)\right|<\infty$. When $a$ is a probability measure, then $T_{y}(a)$ is the stop-loss premium with retention $y$.

Thus we have the stop-loss functional, $T_{y} \circ \Phi: A_{1}^{m} \rightarrow \mathbb{R}$, where

$$
\left(a_{1}, \ldots, a_{m}\right) \mapsto \int_{y}^{\infty} a_{1} * \ldots * a_{m}((x, \infty)) d x .
$$

When $x_{i}=\left(1-q_{i}\right) \delta_{0}+q_{i} y_{i}$ as in $\S 3$, we have that $T_{y} \circ \Phi\left(x_{1}, \ldots, x_{m}\right)$ is $\mathbb{E}\left((S-y)_{+}\right)$ where $S$ is the total claim amount for the individual model. When $\Phi\left(a_{1}, \ldots, a_{m}\right)$ is one of the approximations to this total claim amount distribution given in 
TABLE 6

GERBER PORTFOLIO: ERRORS FOR THE STOP-LOSS APPROXIMATIONS

\begin{tabular}{c|c|c|c|c|c}
\cline { 2 - 6 } & \multicolumn{2}{c|}{ Poisson } & Binomial & \multicolumn{2}{c}{ Negative Binomial } \\
\cline { 2 - 6 } & zeroth & first & zeroth/first & zeroth & first \\
\hline max abs error & 0.0380 & 0.0071 & 0.0069 & 0.0683 & 0.0078 \\
\hline \hline
\end{tabular}

$\S 3$, then the corresponding stop-loss premium $T_{y} \circ \Phi\left(a_{1}, \ldots, a_{m}\right)$ is the resulting zeroth order approximation to the true stop-loss premium.

For a first order approximation, we need the Fréchet derivative of $T_{y}$. It is easy to check that $T_{y}$ is linear and bounded, and that hence $T_{y}$ is Fréchet differentiable at $s$ in $A_{1}$, with derivative $T_{y, s}^{\prime}=T_{y}$ at $s$. Using the chain rule for Fréchet derivatives (Cartan (1971), Theorem 2.2.1 in Chapter 1), we see that the stop-loss functional is Fréchet differentiable at $a=\left(a_{1}, \ldots, a_{m}\right)$ with derivative given by

$$
\left(T_{y} \circ \Phi\right)_{a}^{\prime}(h)=T_{y} \circ \Phi_{a}^{\prime}(h)
$$

where $h=\left(h_{1}, \ldots, h_{m}\right)$. This leads to first order approximations for the stop-loss premium with retention $y$ which are obtained by applying $T_{y}$ to the previous first order approximations for $\Phi\left(x_{1}, \ldots, x_{m}\right)$.

We apply this for the Gerber portfolio of 31 policies. Table 6 gives the maximum absolute error for the stop-loss approximations for retention values $y=0$, ..., 50 .

\section{Miscellaneous REMARKS}

1. The first order approximations (3.1) and (3.3) may be relevant for other areas of applied probability where sums of independent random variables are of interest. In the insurance context, the functional approach is not limited to the specific approximations given in $\$ 3$. For example, an even simpler and cruder approximation is obtained if we rescale the claim sizes so that one unit is $\mathbb{E}(S) / m$ and so $\mathbb{E}(S)=m$. Taking $a_{i}=\delta_{1}, i=1, \ldots, m$, gives a zeroth order approximation $\delta_{m}$ for the distribution of $S$. From (3.3), the first order approximation is

$$
s \approx\left(\sum_{i=1}^{m} x_{i}\right) * \delta_{m-1}-(m-1) \delta_{m}
$$

2. Error bounds are an important aspect of the literature on approximations for the individual risk model. The functional approach does indeed give rise to error bounds on the zeroth order approximations via the Mean Value Theorem (see Proposition 3.3.1 of Chapter 1 in Cartan (1971)). Suppose that $a=\left(a_{1}, \ldots, a_{m}\right)$ and $x=\left(x_{1}, \ldots, x_{m}\right)$ are in $A^{m}$, and that the $a_{i}$ 's and the $x_{i}$ 's are probability 
distributions (as they are in our applications). Then the Mean Value Theorem error bound for using $\Phi(a)=a_{1} * \ldots * a_{m}$ as an approximation for $\Phi(x)=x_{1} *$ $\ldots * x_{m}$ implies

$$
\|\Phi(x)-\Phi(a)\| \leq \sum_{i=1}^{m}\left\|x_{i}-a_{i}\right\|
$$

so that the bound is not new, but recovers an existing known bound, see Feller (1971), page 286, and also Dhaene and Sundt (1997).

3. Higher order derivatives give rise to higher order approximations. Consider a general map $\Psi: U \subseteq B_{1} \rightarrow B_{2}$ where $U$ is an open subset of $B_{1}$ and $\left(B_{i},\|\cdot\|_{i}\right)$, $i=1,2$, are Banach spaces. The second derivative of $\Psi$ at $a$ is a bounded bilinear map $\Psi_{a}^{\prime \prime}(\cdot, \cdot)$ from $B_{1} \times B_{1}$ to $B_{2}$, and is identifiable with the derivative of the map $a \mapsto \Psi_{a}^{\prime}, U \rightarrow \mathcal{L}\left(B_{1}, B_{2}\right)$, where $\mathcal{L}\left(B_{1}, B_{2}\right)$ denotes the set of bounded linear operators from $B_{1}$ to $B_{2}$ ( $\$ 5.1$ in Chapter 1 of Cartan (1971)). For our convolution product map $\Phi$, this leads to a second order approximation

$$
\Phi(x)=\Phi(a)+\Phi_{a}^{\prime}(x-a)+\frac{1}{2} \Phi_{a}^{\prime \prime}(x-a, x-a),
$$

see Theorem 5.6.2 in Cartan (1971). Following a similar approach to that of Proposition 2, we find that

$$
\Phi_{a}^{\prime \prime}(x-a, x-a)=\sum_{i \neq j}\left(x_{i}-a_{i}\right) *\left(x_{j}-a_{j}\right) * \prod_{k \neq i, j} a_{k},
$$

for $a=\left(a_{1}, \ldots, a_{m}\right)$ and $x=\left(x_{1}, \ldots, x_{m}\right)$ in $A^{m}$. For the stop-loss functional, we use the fact that $\left(T_{y} \circ \Phi\right)_{a}^{\prime}(x)=T_{y}\left(\Phi_{a}^{\prime}(x)\right)$ (see $\left.\S 5\right)$, and that $T_{y}$ is linear and bounded, to see that

$$
\left(T_{y} \circ \Phi\right)_{a}^{\prime \prime}(x-a, x-a)=T_{y}\left(\sum_{i \neq j}\left(x_{i}-a_{i}\right) *\left(x_{j}-a_{j}\right) * \prod_{k \neq i, j} a_{k}\right) .
$$

Even when $a_{1}=\ldots=a_{m}$, both these second derivatives still involve the sum $\sum_{i \neq j} x_{i} * x_{j}$. For calculation via fast Fourier transform, in general this gives no simplification over direct calculation of $x_{1} * \ldots * x_{m}$. For higher order approximations, the computational burden increases. Thus the main practical gain of the present approach is achieved by the first order approximations arising from the first derivative, and there seems little further benefit from going on to higher order approximations beyond the first.

4. The second derivative also provides an upper bound on the $\|\cdot\|$-error of the first order approximations. This bound is given by

$$
\left\|\Phi(x)-\Phi(a)-\Phi_{a}^{\prime}(x-a)\right\| \leq \frac{1}{2} \sup _{0 \leq t \leq 1}\left\|\Phi_{(1-t) a+t x}^{\prime \prime}\right\|\|x-a\|^{2},
$$


where $a=\left(a_{1}, \ldots, a_{m}\right)$ and $x=\left(x_{1}, \ldots, x_{m}\right)$ are in $A^{m}$ (Theorem 5.6.2 in Chapter 1 of Cartan (1971)). If the $a_{i}$ 's and $x_{i}$ 's are all probability distributions, then $\sup _{0 \leq t \leq 1}\left\|\Phi_{(1-t) a+t x}^{\prime \prime}\right\| \leq 1$, and so we obtain the following bound on the error for the new first order approximation to $\Phi(x)$

$$
\left\|\Phi(x)-\Phi(a)-\Phi_{a}^{\prime}(x-a)\right\| \leq \frac{1}{2}\|x-a\|^{2}=\frac{1}{2}\left(\sum_{i=1}^{m}\left\|x_{i}-a_{i}\right\|\right)^{2} .
$$

For the stop-loss functional, we find

$$
\left\|T_{y} \circ \Phi(x)-T_{y} \circ \Phi(a)-\left(T_{y} \circ \Phi\right)_{a}^{\prime}(x-a)\right\| \leq \frac{1}{2}\left\|T_{y}\right\|\|x-a\|^{2} \leq \frac{1}{2}\left(\sum_{i=1}^{m}\left\|x_{i}-a_{i}\right\|\right)^{2},
$$

because $\left\|T_{y}\right\| \leq 1$.

5. The error bounds in Remarks 2 and 4 above are not necessarily the best bounds that can be achieved in particular cases. Together, (6.1) and (6.2) tell us what we might have expected: if $x_{i}$ is sufficiently close to $a_{i}$ for each $i$, then the zeroth order approximation is close to the true distribution, and the first order approximation is even closer. However, these bounds do not preclude the possibility of good zeroth and first order approximations even when the righthand sides of (6.1) and (6.2) are not small. Different approaches from the functional approach considered here may well give rise to better error bounds. Nevertheless, the numerical examples in $\S \S 4$ and 5 demonstrate that the functional approach can provide useful and relatively easily calculated approximations that are improvements upon the known zeroth order approximations in the compound Poisson, the compound negative binomial, and in the normal case.

\section{ACKNOWLEDGEMENTS}

I would like to thank the referees for their helpful comments, which have led to improvements in both the content and presentation of the paper.

\section{REFERENCES}

Cartan, H. (1971) Differential Calculus. Hermann, Paris and Kershaw, London.

ČEKANAVIČIUS, V., and WANG, Y.H. (2003) Compound Poisson approximation for sums of discrete random variables. Adv. Appl. Prob. 35, 228-250.

CHARLES, D. (2002) A comparison of possible methods for approximating aggregate claims probabilities of the individual risk model. Unpublished MPhil thesis, University of Cambridge.

CrAmÉr. H. (1930) On the mathematical theory of risk. Forsäkringtiebolaget Skandia 1855-1930, Parts I and II, Stockholm, 1930, 7-84, In Harald Cramér: Collected Works, Vol I, ed: A. Martin-Löf, Springer-Verlag, 1994.

De PRIL, N. (1986) On the exact computation of the aggregate claims distribution in the individual life model. ASTIN Bulletin 16, 109-112.

De PRIL, N. (1989) The aggregate claims distribution in the individual model with arbitrary positive claims. ASTIN Bulletin 19, 9-24. 
De Pril, N., and Dhaene, J. (1992) Error bounds for compound Poisson approximations of the individual risk model. ASTIN Bulletin 22, 135-148.

DhAenE, J., and SundT, B. (1997) On error bounds for approximations to aggregate claims distributions. ASTIN Bulletin 27, 243-262.

Dunford, N. and Shwartz, J.T. (1958) Linear Operators: Part I, General Theory. Interscience, New York.

Embrechts, P., Grübel, R. and Pitts, S.M. (1993) Some applications of the fast Fourier transform algorithm in insurance mathematics. Statistica Neerlandica 47, 59-75.

Feller, W. (1971) An Introduction to Probability Theory and Its Applications. Vol. 2, 2nd Ed. Wiley, New York.

Gerber, H.U. (1979) An Introduction to Mathematical Risk Theory. Huebner Foundation for Insurance Education, Philadelphia.

GRÜBEL, R. (1989) Stochastic models as functionals: some remarks on the renewal case. J. Appl. Prob. 26, 296-303.

GrüBeL, R. and PitTs, S.M. (1992) A functional approach to the stationary waiting time and idle period distributions of the GI/G/1 queue. Ann. Probab. 20, 1754-1778.

Hipp, C. (1986) Improved approximations for the aggregate claims distribution in the individual model. ASTIN Bulletin 16, 89-100.

Klugman, S.A, Panjer, H.H., and Willmot, G.E. (1998) Loss Models: from Data to Decisions. Wiley, New York.

KoRNYA, P.S. (1983) Distribution of aggregate claims in the individual risk theory model. Transactions of the Society of Actuaries 35, 823-836.

Kuon, S., RadtKe, M., and ReIch, A. (1993) An appropriate way to switch from the individual risk model to the collective one. ASTIN Bulletin 23, 23-54.

PANJER, H.H. (1981) Recursive evaluation of a family of compound distributions. ASTIN Bulletin 12, 22-26.

PitTs, S.M. (1998) A functional approach to approximations for the observed open times in single ion channels. Journal of Mathematical Analysis and Applications 219, 260-278.

PitTs, S.M. (1999) Approximations for semi-Markov single ion channel models. In Semi-Markov Models and Applications. eds J. Janssen and N. Limnios. Kluwer, Dordrecht. 103-115.

Politis, K. and PITTS, S.M. (1998) Approximations for the solutions of renewal-type equations. Stochastic Processes and their Applications 78, 195-216.

Rolski, T., Schmidli, H., Schmidt, V., and Teugels, J. (1999) Stochastic Processes for Insurance and Finance. Wiley, Chichester.

Rudin, W. (1986) Real and Complex Analysis. (3rd ed.) McGraw-Hill, New York.

\section{SUSAN M. PITTS}

University of Cambridge 